\title{
Vítimas de causas externas alcoolizadas atendidas em serviço de urgência e emergência: estudo seccional*
}

\author{
Cosma Rosário Glória Santos Aguiar ${ }^{1}$, Rafael Tavares Jomar², \\ Ângela Maria Mendes Abreu ${ }^{3}$, Teresa Maria Mendes Diniz de Andrade Barroso ${ }^{4}$
}

\footnotetext{
* Extraído da Dissertação de Mestrado “Acidentes e violências relacionadas com o consumo de álcool: perfil das vitimas atendidas no serviço de urgência do Hospital Central de São Tomé" defendida, em 2015, no Programa de Pós-Graduação em Enfermagem de Saúde Mental e Psiquiatria da Escola Superior de Enfermagem de Coimbra.

${ }^{1}$ Enfermeira, Mestre em Enfermagem de Saúde Mental e Psiquiatra. Enfermeira do Hospital Dr. Ayres de Menezes. São Tomé, República Democrática de São Tomé e Príncipe. E-mail:

aguiarcosma@yahoo.com.br.

${ }^{2}$ Enfermeiro, Doutor em Saúde Coletiva. Professor Assistente da Faculdade de Enfermagem da Universidade do Estado do Rio de Janeiro. Rio de Janeiro, RJ, Brasil. Email: rafaeljomar@yahoo.com.br.

${ }^{3}$ Enfermeira, Doutora em Enfermagem. Professor Associado da Escola de Enfermagem Anna Nery da Universidade Federal do Rio de Janeiro. Rio de Janeiro, RJ, Brasil. E-mail: angelabreu@globo.com.

${ }^{4}$ Enfermeira, Doutora em Enfermagem. Professor Adjunto da Escola Superior de Enfermagem de Coimbra. Coimbra, Portugal. E-mail: tbarroso@esenfc.pt.
}

Recebido: 05/02/2017.

Aceito: 06/06/2017.

Publicado: $24 / 11 / 2017$.

Como citar esse artigo:

Aguiar CRGS, Jomar RT, Abreu AMM, Barroso TMMDA. Vítimas de causas externas alcoolizadas atendidas em serviço de urgência e emergência: estudo seccional. Rev. Eletr. Enf. [Internet]. 2017 [acesso em:_____];19:a32. Disponível em:

http://dx.doi.org/10.5216/ree.v19.45271.

\section{RESUMO}

Estudo seccional que objetivou caracterizar o atendimento e o perfil de vítimas de causas externas alcoolizadas no principal serviço de urgência e emergência de São Tomé e Príncipe. Para tanto, todas as 672 vítimas atendidas entre abril de 2014 e abril de 2015 que estavam sob efeito do álcool tiveram seus prontuários avaliados. A maioria delas era jovem $(56,8 \%)$, do sexo masculino $(76,5 \%)$, cujos atendimentos foram realizados majoritariamente no período noturno $(38,8 \%)$ dos finais de semana $(75,1 \%)$ e resultaram em alta hospitalar (62,9\%). Quanto ao tipo de causa externa, as agressões foram aquelas que demandaram mais atendimentos $(40,0 \%)$, seguidas pelos acidentes de trabalho ou de trajeto $(30,5 \%)$ e dos acidentes de trânsito $(29,1 \%)$. Os resultados deste estudo pioneiro em São Tomé e Príncipe apontam as causas externas, especialmente as agressões, como um grave problema de saúde pública no país que demanda muitos atendimentos hospitalares.

Descritores: Consumo de Bebidas Alcoólicas; Causas Externas; Serviços Médicos de Emergência.

\section{INTRODUÇÃO}

As condições de saúde dos indivíduos e das populações são direta e indiretamente afetadas pelas causas externas, que reduzem os anos de vida produtiva, geram lesões e sequelas - por vezes irreversíveis - e produzem elevado número de mortes ${ }^{(1)}$. Causas externas são traumatismos, lesões ou quaisquer outros agravos à saúde intencionais ou não - de início súbito como consequência imediata de 
violência ou outra causa exógena. Neste grupo, incluem-se as lesões provocadas por eventos no transporte, homicídios, agressões, quedas, afogamentos, envenenamentos, suicídios, queimaduras, lesões por deslizamento ou enchente e outras ocorrências provocadas por circunstâncias ambientais ${ }^{(2)}$.

Segundo a Organização Mundial da Saúde (OMS), o consumo de álcool está relacionado a 3,3 milhões de mortes a cada ano. Assim, quase $6 \%$ das mortes no planeta são atribuídas total ou parcialmente ao consumo dessa substância ${ }^{(3)}$, apontada como o principal fator de risco para a alta morbimortalidade provocada pelas causas externas ${ }^{(4-6)}$.

Globalmente, estima-se que indivíduos com idade de 15 anos ou mais consumiram em torno de 6,2 litros de álcool puro em 2010. Na República Democrática de São Tomé e Príncipe, país africano de língua oficial portuguesa, o consumo total de álcool puro estimado para o mesmo ano foi equivalente a 7,1 litros por pessoa, quantidade superior à média mundial. De todas as mortes registradas neste país, em 2010, a OMS estima que 3,5\% podem ser atribuídas ao consumo de álcool, percentual maior que o estimado para todo o continente africano $(3,3 \%)^{(3)}$.

Em extensa revisão de literatura efetuada pelos autores, não foram encontrados estudos sobre o atendimento de vítimas de causas externas alcoolizadas em serviços hospitalares da República Democrática de São Tomé e Príncipe. Com o intuito de preencher a lacuna existente no conhecimento do perfil dessas vítimas no país e, assim, colaborar para a formulação de estratégias de prevenção de causas externas para a população geral e grupos específicos, o presente estudo foi conduzido com o objetivo de caracterizar o atendimento e o perfil de vítimas de causas externas alcoolizadas no principal serviço de urgência e emergência de São Tomé e Príncipe.

\section{MÉTODOS}

Estudo descritivo de caráter seccional conduzido em unidade hopitalar de referência para atendimento de urgências e emergências localizado em São Tomé, que pertence à República Democrática de São Tomé e Príncipe - um país insular com pouco mais de 190.000 habitantes.

Entre os meses de abril de 2014 e abril de 2015, foram realizados 34.600 atendimentos no cenário do estudo, dos quais 4.277 foram de vítimas de causas externas. A amostra do presente estudo foi composta por todas as 672 vítimas de causas externas que, a despeito da idade, segundo registro em prontuário, estavam sob efeito do álcool - único critério de elegibilidade adotado pelo presente estudo.

Em julho de 2015, uma enfermeira devidamente treinada consultou os prontuários das 672 vítimas de causas externas alcoolizadas, de onde foram coletadas e transcritas para formulário previamente elaborado pelos autores informações sociodemográficas (sexo, idade, cor da pele e situação conjugal), características cronológicas relativas a seu atendimento (hora, dia da semana e mês), ao tipo de causa externa em que estava envolvida (agressão, acidente de trânsito ou acidente de trabalho ou de trajeto ${ }^{1}$ ) e ao desfecho

\footnotetext{
${ }^{1}$ Acidente de trajeto é aquele que ocorre no trajeto da residência para o trabalho ou do trabalho para a residência. 
hospitalar nas 24 horas seguintes ao atendimento (alta, internação, cirurgia ou óbito).

A análise de dados foi feita no software SPSS versão 19.0 com distribuição de frequências simples.

A condução do estudo foi aprovada pela Comissão de Ética em Pesquisa da Unidade de Investigação em Ciências de Saúde: Enfermagem da Escola Superior de Enfermagem de Coimbra, sob o no de protocolo 284/05-2015.

\section{RESULTADOS}

As vítimas tinham média de idade de 30,5 anos (DP: 11,6), eram predominantemente do sexo masculino $(76,5 \%)$ - três homens para cada mulher - casadas ou em união estável $(79,5 \%)$ e de cor da pele preta $(99,0 \%)$ (Tabela 1$)$.

Tabela 1: Características sociodemográficas das vítimas de causas externas alcoolizadas $(n=672)$. São Tomé, República Democrática de São Tomé e Príncipe, 2014/2015.

\begin{tabular}{|c|c|c|}
\hline Variáveis & $\mathbf{n}$ & $\%$ \\
\hline \multicolumn{3}{|l|}{ Sexo } \\
\hline Masculino & 514 & 76,5 \\
\hline Feminino & 158 & 23,5 \\
\hline \multicolumn{3}{|l|}{ Faixa etária } \\
\hline 15-29 anos & 382 & 56,8 \\
\hline $30-59$ anos & 271 & 40,3 \\
\hline $60-74$ anos & 19 & 2,8 \\
\hline \multicolumn{3}{|l|}{ Cor da pele } \\
\hline Preta & 665 & 99,0 \\
\hline Branca & 07 & 1,0 \\
\hline \multicolumn{3}{|l|}{ Situação conjugal } \\
\hline Casado/Vive em união & 534 & 79,5 \\
\hline Solteiro & 129 & 19,2 \\
\hline Divorciado/Separado & 06 & 0,9 \\
\hline Viúvo & 03 & 0,4 \\
\hline
\end{tabular}

Em relação às características do atendimento, $35 \%$ das vítimas foi atendida entre os meses de junho e agosto, $75,1 \%$ de sexta a domingo e $38,8 \%$ entre 18 e 24 horas (Tabela 2 ).

$\mathrm{Na}$ Tabela 3, podem ser observadas algumas características das causas externas: $40 \%$ foram devidas à agressão e $29,5 \%$ a acidentes de trânsito, dos quais $81 \%$ envolveram motocicletas. Nas primeiras 24 horas após o atendimento hospitalar, 62,9\% das vítimas recebeu alta e 5,1\% evoluiu para óbito. 
Tabela 2: Características cronológicas do atendimento das vítimas de causas externas alcoolizadas $(n=672)$. São Tomé, República Democrática de São Tomé e Príncipe, 2014/2015.

\begin{tabular}{|c|c|c|}
\hline Variáveis & $\mathbf{n}$ & $\%$ \\
\hline \multicolumn{3}{|l|}{ Meses } \\
\hline Dezembro/Janeiro/Fevereiro & 141 & 20,8 \\
\hline Março/Abril*/Maio & 175 & 26,1 \\
\hline Junho/Julho/Agosto & 236 & 35,2 \\
\hline Setembro/Outubro/Novembro & 120 & 17,8 \\
\hline \multicolumn{3}{|l|}{ Dia da semana } \\
\hline Sexta/Sábado/Domingo & 505 & 75,1 \\
\hline Segunda/Terça/Quarta/Quinta & 167 & 24,9 \\
\hline \multicolumn{3}{|l|}{ Horário (em horas) } \\
\hline $06-12$ & 112 & 16,7 \\
\hline $12-18$ & 196 & 29,2 \\
\hline $18-24$ & 261 & 38,8 \\
\hline $24-06$ & 103 & 15,3 \\
\hline
\end{tabular}

* Abril de 2014 e 2015

Tabela 3: Características e desfecho do atendimento das vítimas de causas externas alcoolizadas $(n=672)$. São Tomé, República Democrática de São Tomé e Príncipe, 2014/2015.

\begin{tabular}{|c|c|c|}
\hline Variáveis & $\mathbf{n}$ & $\%$ \\
\hline \multicolumn{3}{|l|}{ Tipo de acidente } \\
\hline Agressão & 269 & 40,0 \\
\hline Acidente de trabalho ou de trajeto & 205 & 30,5 \\
\hline Acidente de trânsito & 195 & 29,1 \\
\hline Outros & 03 & 0,4 \\
\hline Total & 672 & 100,0 \\
\hline \multicolumn{3}{|l|}{ Veículo envolvido no acidente de trânsito } \\
\hline Motocicleta & 158 & 81,0 \\
\hline Carro & 27 & 13,9 \\
\hline Bicicleta & 10 & 5,1 \\
\hline Total & 195 & 100,0 \\
\hline \multicolumn{3}{|l|}{ Desfecho hospitalar nas primeiras 24 horas } \\
\hline Alta & 423 & 62,9 \\
\hline Internação & 205 & 30,5 \\
\hline Cirurgia & 10 & 1,5 \\
\hline Óbito & 34 & 5,1 \\
\hline Total & 672 & 100,0 \\
\hline
\end{tabular}

\section{DISCUSSÃO}

O presente estudo aponta que a maioria das vítimas de causas externas alcoolizadas atendidas no principal serviço de urgência e emergência de São Tomé era jovem, do sexo masculino, cujos atendimentos foram realizados majoritariamente no período noturno dos finais de semana e resultaram em alta hospitalar.

Resultados semelhantes foram relatados por estudo que descreveu o perfil e os fatores relacionados ao consumo de álcool em atendimentos de emergência por causas externas nas 24 capitais brasileiras e no Distrito Federal em 2011, onde a maioria dos atendimentos foi realizado a noite e nos finais de semana. Além disso, o consumo de álcool foi significativamente superior entre as vítimas do sexo masculino com idades entre 18 e $39 \operatorname{anos}^{(7)}$.

As causas externas constituem um importante fator de morbimortalidade entre homens jovens, contribuindo fortemente para a redução da expectativa dessa população ${ }^{(8)}$. Cerca de $48 \%$ dos habitantes da 
República Democrática de São Tomé e Príncipe são do sexo masculino, em sua maioria, chefes de famílias

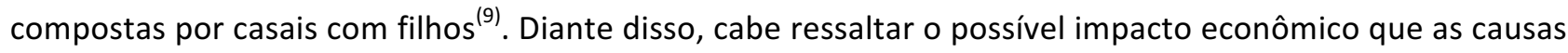
externas provocaram nas famílias das 514 (76,5\%) vítimas do sexo masculino alcoolizadas que foram atendidas no principal serviço de urgência e emergência de São Tomé no período estudado.

A ocorrência mais frequente de atendimentos de vítimas de causas externas alcoolizadas nos finais de semana e a noite é compatível com a literatura científica ${ }^{(7,10)}$, possivelmente, porque nessas ocasiões são mais comuns as aglomerações de pessoas em festas e celebrações, onde o consumo de álcool é maior e, consequentemente, maior o risco de ocorrência de causas externas ${ }^{(10)}$.

No que diz respeito aos meses de atendimento das vítimas, a maioria deles ocorreu entre junho e agosto. Isto pode estar relacionado ao fato de muitas festas religiosas de santos populares acontecerem nesses meses, à existência de um feriado nacional em julho e à Gravana - período de estação seca que coincide com meses de férias e turismo em São Tomé. Logo, os autores acreditam que a aglomeração de pessoas e os festejos no período possam ter influenciado o maior número de atendimentos de vítimas de causas externas sob efeito do álcool.

Tal como encontrado por estudo que descreveu o perfil das vítimas de causas externas atendidas em serviços selecionados de urgência e emergência do Sistema Único de Saúde do Brasil em $2011^{(7)}$, a alta hospitalar foi o desfecho mais frequente das vítimas alcoolizadas atendidas no principal serviço de urgência e emergência de São Tomé. Cabe destacar, entretanto, que 5,1\% delas evoluiu para óbito nas 24 horas seguintes ao atendimento.

A OMS estima que, em todo o mundo, ocorram cerca de 3,3 milhões de mortes a cada ano em decorrência do consumo nocivo de álcool - um dos fatores de risco de maior impacto para a morbidade e incapacidades. Além disso, quase $6 \%$ de todas as mortes no mundo são atribuídas total ou parcialmente ao consumo dessa substância ${ }^{(3)}$. Mesmo assim, a maioria dos povos e culturas aceita e incentiva o consumo de álcool, associando-o a celebrações sociais e cerimônias religiosas.

Convém destacar que a diminuição do consumo de álcool constitui uma prioridade de saúde pública, uma vez que impacta não apenas na redução da morbimortalidade por causas externas, mas também por doenças crônicas não transmissíveis. A diminuição do consumo dessa substância depende principalmente de medidas de regulação adotadas pelos governos, bem como do enfrentamento do poder das indústrias, como o ajuste monetário das bebidas alcóolicas, que poderia torná-las mais caras e, consequentemente, menos disponíveis ${ }^{(11)}$.

No que tange ao tipo de causa externa sofrida pelas vítimas, as agressões foram aquelas que demandaram mais atendimentos, seguidas pelos acidentes de trabalho ou de trajeto e dos acidentes de trânsito. Estudo descritivo sobre o perfil das vítimas de causas externas atendidas em serviços brasileiros de urgência e emergência relatou resultados diferentes: quedas, acidentes de trânsito e agressões foram, respectivamente, os motivos de atendimentos mais frequentes ${ }^{(7)}$. As quedas estarem entre os motivos mais frequentes de atendimentos de vítimas de causas externas no estudo citado, possivelmente, se deve ao 
expressivo percentual de idosos atendidos $(8,3 \% ; n=3.308)^{(7)}$, que é 2,9 vezes maior que o percentual de idosos do presente estudo $(2,8 \% ; n=19)$.

Segundo a OMS, o consumo de álcool está mais fortemente associado a um comportamento agressivo do que ao uso de qualquer outra substância psicotrópica ${ }^{(12)}$. Estudos prévios evidenciaram que o consumo de álcool desempenha papel decisivo em aproximadamente metade dos crimes violentos ${ }^{(13)}$ e agressões sexuais ${ }^{(14)}$ em todo o mundo. Além disso, há evidências de que altos níveis de impulsividade ${ }^{(15)} \mathrm{e}$ baixa tolerância ao estresse ${ }^{(16)}$ podem reforçar a inclinação para se comportar agressivamente sob a influência dessa substância.

Embora as agressões - ou violência interpessoal - constituam um fenômeno universal que tem se apresentado como habitual na sociedade contemporânea, são escassos os estudos que investigam casos de atendimento por essa causa em serviços de urgência e emergência ${ }^{(17)}$. Já os atendimentos de vítimas de acidentes de trânsito em serviços de urgência e emergência têm sido bem descritos pela literatura científica ${ }^{(5-}$ $7,18)$

No tocante ao envolvimento do álcool em acidentes de trânsito, sabe-se que sua presença no organismo produz diversas alterações neuromotoras. Mesmo em concentrações mais baixas, a presença do álcool resulta em diminuição da atenção, falsa percepção da velocidade, euforia e dificuldade de discernir luminosidades distintas. Concentrações mais altas prejudicam progressivamente a tomada de decisão, interferindo nos reflexos e no comportamento, podendo levar à embriaguez, ao torpor e ao coma alcoólico $^{(19)}$.

Neste estudo, $29,1 \%$ dos atendimentos de vítimas alcoolizadas foram devidos aos acidentes de trânsito, dos quais $81 \%$ tiveram o envolvimento de motocicletas. Estudo que descreveu as características de motociclistas envolvidos em acidentes de trânsito atendidos em serviços públicos de urgência e emergência nas capitais brasileiras e Distrito Federal em 2014, apontou que 13,3\% das vítimas fizeram referência ao uso de álcool nas seis horas anteriores ao acidente ${ }^{(20)}$.

Na República Democrática de São Tomé e Príncipe, acidentes de trânsito envolvendo motocicletas figuram entre as primeiras causas de morte. A profissão de "moto-taxista" é relativamente recente, porém uma das que mais cresce no país. Seus profissionais são majoritariamente jovens e muitos não estão habilitados para conduzir veículo automotor. Por isso, acredita-se que imprudência e falta de habilitação sejam as principais causas de acidentes de trânsito no país ${ }^{(21)}$.

As motocicletas são consideradas uma das formas mais perigosas de transporte motorizado devido ao pequeno tamanho e à exposição direta ao impacto, o que torna seus ocupantes mais vulneráveis à traumas múltiplos e de maior gravidade ${ }^{(22)}$. Cabe ressaltar que, em acidentes de trânsito, motociclistas tem risco de morte 30 vezes maior, quando comparados a ocupantes de outros tipos de veículos motorizados ${ }^{(23)}$.

Para minimizar o risco de morte dessa classe trabalhadora emergente no país, recomendam-se a manutenção de um bom estado de conservação das vias e rodovias e a fiscalização sistemática de obediência às leis de trânsito, especialmente, no que se refere ao uso de equipamentos de proteção individual, como os 
capacetes $^{(24)}$.

$\mathrm{Na}$ extensa revisão de literatura efetuada pelos autores, não foram encontrados estudos que tenham investigado o atendimento de vítimas de causas externas alcoolizadas em serviços hospitalares de urgência e emergência da República Democrática de São Tomé e Príncipe, o que não permitiu comparações de nossos resultados. Por isso, acreditamos que este seja o primeiro estudo com esse fim conduzido no país. Outras investigações sobre o tema, portanto, são necessárias para colaborar com a formulação de estratégias de prevenção de causas externas no país, especialmente, investigações sobre vítimas de agressões atendidas em serviços de urgência e emergência.

Dentre suas limitações, apontamos seu desenvolvimento com dados secundários registrados com finalidade basicamente assistencial e administrativa, sem fins de pesquisa. Outra limitação refere-se à diversidade de registros sobre o consumo de álcool nos prontuários das vítimas ("hálito alcoólico", "hálito alcoólico intenso", "hálito etílico", "paciente alcoolizado", "paciente sob efeito do álcool”, "apresenta cheiro intenso de álcool", dentre outros), o que impediu a classificação das vítimas em níveis de alcoolização, já que não havia padronização dos registros, tampouco resultados de alcoolemia. Ressaltamos também a possibilidade de outros atendimentos à vítimas de causas externas alcoolizadas terem sido efetuados no período, mas que não puderam ser considerados pelo estudo por conta da ausência de registro em prontuário.

\section{CONCLUSÃO}

Em suma, os resultados deste estudo pioneiro apontam que a maioria das vítimas de causas externas alcoolizadas atendidas no principal serviço de urgência e emergência de São Tomé e Príncipe era jovem (56,8\%), do sexo masculino $(76,5 \%)$, cujos atendimentos foram realizados majoritariamente no período noturno $(38,8 \%)$ dos finais de semana $(75,1 \%)$ e resultaram em alta hospitalar $(62,9 \%)$. Assim, as causas externas envolvendo o uso de álcool, especialmente as agressões, responsáveis por 40,0\% dos atendimentos das vítimas, constituem um grave problema de saúde pública no país por demandarem muitos atendimentos hospitalares.

\section{REFERÊNCIAS}

1. Ribeiro AP, Souza ER, Bahia CA. Accidental events: challenges for public policy, surveillance and prevention. Cien Saude Colet [Internet]. 2016 [acesso em: 2017 jan 06];21(12):3646-46. Disponível em: http://www.scielo.br/pdf/csc/v21n12/en_1413-8123-csc-21-12-3646.pdf.

2. Gonsaga RAT, Rimoli CF, Pires EA, Zogheib FS, Fujino MVT, Cunha MB. Evaluation of the mortality due to external causes. Rev Col Bras Cir [Internet]. 2012 [acesso em: 2017 jan 06];39(4):263-67. Disponível em:

http://www.scielo.br/pdf/rcbc/v39n4/en_04.pdf.

3. World Health Organization. Global status report on alcohol and health - 2014 [Internet]. Geneva: WHO; 2014 [acesso em: 2017 abr 20]. Disponível em:

http://www.who.int/substance_abuse/publications/global_alcohol_report/en/. 
4. Cruz CC, Sarcinelli PN, Silva JJO, Meyer TSM, Bochner R, Alves SR. Causas externas relacionadas à alcoolemia registradas pelo Instituto Médico Legal no município do Rio de Janeiro. Cad saúde colet [Internet]. 2014 [acesso em: 2017 jan 06];22(1):8-15. Disponível em: http://www.scielo.br/pdf/cadsc/v22n1/1414-462X-cadsc-22-01-00008.pdf. 5. Mascarenhas MDM, Neves ACM, Monteiro RA, Silva MMA, Malta DC. Emergency room visits due to external causes and alcohol consumption - Capitals and the Federal District, Brazil, 2011. Cien Saude Colet [Internet]. 2015 [acesso em: 2017 jan 06];20(4):1037-46. Disponível em: http://www.scielo.br/pdf/csc/v20n4/1413-8123-csc-20-04-01037.pdf. 6. Korcha R, Cherpitel CJ, Yu Y, Bond J, Andreuccetti G, Borges G, Bazargn-Hejazi S. Alcohol use and injury severity among emergency department patients in six countries. J Addict Nurs [Internet]. 2013 [acesso em: 2017 jan 06];24(3):158-65. Disponível em:

http://journals.Iww.com/jan/pages/articleviewer.aspx?year=2013\&issue=06000\&article=00005\&type=abstract.

7. Neves ACM, Mascarenhas MDM, Silva MMA, Malta DC. Perfil das vítimas de violências e acidentes atendidas em serviços de urgência e emergência do Sistema Único de Saúde em capitais brasileiras - 2011. Epidemiol Serv Saúde [Internet]. 2013 [acesso em: 2017 jan 07];22(4):587-96. Disponível em:

http://scielo.iec.pa.gov.br/pdf/ess/v22n4/v22n4a05.pdf.

8. Ministério da Saúde (Brasil). Saúde Brasil 2012: uma análise da situação de saúde e dos 40 anos do Programa Nacional de Imunizações [Internet]. Brasília: Ministério da Saúde; 2013 [acesso em: 2017 abr 20]. Disponível em: http://bvsms.saude.gov.br/bvs/publicacoes/saude_brasil_2012 analise situacao saude.pdf.

9. Instituto Nacional de Estatísticas (República Democrática de São Tomé e Príncipe). Inquérito Orçamento Familiar 2010 [Internet]. São Tomé: INE; 2010 [acesso em: 2017 abr 20]. Disponível em:

http://www.ine.st/Documentacao/IOF\%202009/survey0/index.html.

10. World Health Organization. Beber e dirigir: manual de segurança no trânsito para profissionais de trânsito e saúde [Internet]. Genebra: WHO; 2007 [acesso em: 2017 abr 20]. Disponível em:

http://www.who.int/roadsafety/publications/DrinkingAndDrivingManual_POR.pdf?ua=1.

11. Garcia LP, Freitas LRS. Heavy drinking in Brazil: results from the 2013 National Health Survey. Epidemiol Serv Saúde [Internet]. 2015 [acesso em: 2017 abr 20];24(2):227-37. Disponível em:

http://www.scielo.br/pdf/ress/v24n2/en_2237-9622-ress-24-02-00227.pdf.

12. World Health Organization. World Health Organization expert committee on problems related to alcohol consumption [Internet]. Geneva: WHO; 2007 [acesso em: 2017 abr 20]. Disponível em:

http://www.who.int/substance_abuse/expert_committee_alcohol/en/.

13. Darke S. The toxicology of homicide offenders and victims: a review. Drug Alcohol Rev [Internet]. 2010 [acesso em: 2017 abr 20];29(2):202-15. Disponível em: http://onlinelibrary.wiley.com/wol1/doi/10.1111/j.1465-

3362.2009.00099.x/abstract.

14. Testa M. The impact of men's alcohol consumption on perpetration of sexual aggression. Clin Psychol Rev [Internet]. 2002 [acesso em: 2017 abr 20];22(8):1239-63. Disponível em:

https://www.ncbi.nlm.nih.gov/pubmed/12436812.

15. Cunradi CB, Ames GM, Duke M. The relationship of alcohol problems to the risk for unidirectional and bidirectional intimate partner violence among a sample of blue-collar couples. Violence Vict [Internet]. 2011 [acesso em: $2017 \mathrm{abr}$ 20];26(2):147-58. Disponível em: https://www.ncbi.nlm.nih.gov/pmc/articles/PMC3579767/.

16. Wray TB, Simons JS, Dvorak RD, Gaher RM. Trait-based affective processes in alcohol-involved "risk behaviors". Addict Behav [Internet]. 2012 [acesso em: 2017 abr 20];37(11):1230-39. Disponível em:

https://www.ncbi.nlm.nih.gov/pmc/articles/PMC3401606/.

17. Cecilio LPP, Garbin CAS, Rovida TAS, Queiróz APDG, Garbin AJI. Violência interpessoal: estudo descritivo dos casos não fatais atendidos em uma unidade de urgência e emergência referência de sete municípios do estado de São Paulo, Brasil, 2008 a 2010. Epidemiol Serv Saúde [Internet]. 2012 [acesso em: 2017 jan 12];21(2):293-304. Disponível em: http://scielo.iec.pa.gov.br/pdf/ess/v21n2/v21n2a12.pdf.

18. Oliveira APP, Abreu AMM, Paixão LAR, Faria VS. Possível impacto da "Lei Seca" nos atendimentos a vítimas de acidentes de trânsito em uma unidade de emergência. Esc Anna Nery [Internet]. 2013 [acesso em: 2017 jan 12];17(1):54-59. Disponível em: http://www.scielo.br/pdf/ean/v17n1/08.pdf.

19. National Highway Traffic Safety Administration. A review of the literature on the effects of low doses of alcohol on driving-related skills [Internet]. [acesso em: 2017 jan 12]. Disponível em: http://www.nhtsa.dot.gov.

20. Mascarenhas MDM, Souto RMCV, Malta DC, Silva MMA, Lima CM, Montenegro MMS. Characteristics of motorcyclists involved in road traffic accidents attended at public urgent and emergency services. Cien Saude Colet

Rev. Eletr. Enf. [Internet]. 2017 [acesso em:_/_/_];19:a32. Disponivel em: http://dx.doi.org/10.5216/ree.v19.45271. 
[Internet]. 2016 [acesso em: 2017 jan 12];21(12):3661-71. Disponível em:

http://www.scielo.br/pdf/csc/v21n12/en_1413-8123-csc-21-12-3661.pdf.

21. Aumento de acidentes de viação preocupa São Tomé e Príncipe. Deutsche Welle [Internet]. 2013 Set 04 [acesso em: 2017 jan 12]. Disponível em: http://www.dw.com/pt-002/aumento-de-acidentes-de-via\%C3\%A7\%C3\%A3opreocupa-s\%C3\%A3o-tom\%C3\%A9-e-pr\%C3\%ADncipe/a-17067329.

22. Golias ARC, Caetano R. Acidentes entre motocicletas: análise dos casos ocorridos no estado do Paraná entre julho de 2010 e junho de 2011. Cien Saude Colet [Internet]. 2013 [acesso em: 2017 jan 12];18(5):1235-46. Disponível em: http://www.scielo.br/pdf/csc/v18n5/08.pdf.

23. Instituto de Pesquisa Econômica Aplicada. Acidentes de trânsito nas rodovias federais brasileiras: caracterização, tendências e custos para a sociedade - relatório de pesquisa [Internet]. Brasília: IPEA; 2015 [acesso em: 2017 abr 20]. Disponível em:

http://www.ipea.gov.br/portal/images/stories/PDFs/relatoriopesquisa/150922_relatorio_acidentes_transito.pdf. 24. Organização Pan-Americana da Saúde. Capacetes: manual de segurança no trânsito para os gestores e profissionais de saúde [Internet]. Brasília: OPAS; 2007 [acesso em: 2017 abr 20]. Disponível em:

http://apps.who.int/iris/bitstream/10665/43261/4/9241562994_por.pdf. 